\title{
review
}

\section{Mechanical recanalization for acute bilateral cerebral artery occlusion - literature overview with a case}

\author{
Miran Jeromel ${ }^{1,2}$, Zoran V Milosevic ${ }^{1}$, Janja Pretnar Oblak ${ }^{3}$ \\ ${ }^{1}$ Department of Diagnostic and Interventional Neuroradiology, University Medical Centre Ljubljana, Ljubljana, Slovenia \\ 2 Department of Diagnostic and Interventional Radiology, General Hospital Slovenj Gradec, Slovenj Gradec, Slovenia \\ ${ }^{3}$ Department for Vascular Neurology and Intensive Neurological Therapy, University Medical Centre Ljubljana, Ljubljana, \\ Slovenia
}

Radiol Oncol 2020; 54(2): 144-148.

Received 19 January 2020

Accepted 5 March 2020

Correspondence to: Miran Jeromel, M.D., Ph.D., Department of Diagnostic and Interventional Radiology, General Hospital Slovenj Gradec, Gosposvetska cesta 1, SI-2380 Slovenj Gradec, Slovenia. E-mail: miran.jeromel@gmail.com

Disclosure: No potential conflicts of interest were disclosed.

\begin{abstract}
Background. Acute bilateral internal carotid artery (ICA) and/or middle cerebral artery (MCA) occlusion is extremely rare and associated with poor clinical outcomes. There are only a few reports in the literature about mechanical thrombectomy being performed for acute bilateral occlusions. The treatment strategies and prognoses (clinical outcomes) are therefore unclear.

Methods. A systematic review of the literature was performed through several electronic databases with the following search terms: acute bilateral stroke, mechanical recanalization and thrombectomy.

Results. In the literature, we identified five reports of six patients with bilateral ICA and/or MCA occlusion treated with mechanical recanalization. Additionally, we report our experience with a subsequent contralateral large brain artery occlusion during intravenous thrombolytic therapy, where the outcome after mechanical thrombectomy was not dependent on the time from stroke onset but rather on the capacity of collateral circulation exclusively.

Conclusions. Acute bilateral cerebral (ICA and/or MCA) occlusion leads to sudden severe neurological deficits (comas) with unpredicted prognoses, even when mechanical recanalization is available. As the collateral capacity seems to be more important than the absolute time to flow restoration in determining the outcomes, simultaneous thrombectomy by itself probably does not lead to improved functional outcomes.
\end{abstract}

Key words: acute bilateral stroke; mechanical recanalization; thrombectomy

\section{Introduction}

Acute embolic bilateral internal carotid artery (ICA) and/or middle cerebral artery (MCA) occlusion leads to sudden comas with poor prognoses..$^{1-9}$ The reported incidence of this condition in stroke patients treated with intravenous or intra-arterial therapy is $0.34 \% .{ }^{8}$ There are few reports on the endovascular treatment (mechanical recanalization - thrombectomy) of this rare condition. The treat- ment strategies and prognoses (clinical outcomes) are therefore unclear.

The aim of the present article was to discuss our experience with the treatment of this severe condition through a few published reports. To the best of our knowledge, we report the first case of subsequent contralateral large brain artery occlusion during intravenous thrombolytic therapy, where the outcome after mechanical thrombectomy was not dependent on the time from stroke onset but 
rather on the capacity of collateral circulation exclusively.

\section{Methods}

A systematic review of the literature was performed through several electronic databases:

PubMed (US National Library of Medicine, http://www.ncbi.nlm.nih.gov/pubmed), Google Scholar (https://scholar.google.com/), Scopus (Elsevier, http://www.scopus.com/), DeGruyer (https://www.degruyer.com) and Cochrane Library (http://www.cocranelibrary.com). The following search terms were used: acute bilateral stroke, mechanical recanalization and thrombectomy.

\section{Results}

Altogether, five reports of six patients with bilateral ICA and/or MCA occlusion that met the inclusion criteria were identified (Table 1). Four patients were females (age range 64-78 years), one was male (72 years), and one was a middle-aged patient of an undetermined sex. All patients were treated with mechanical recanalization (thrombectomy) using different endovascular techniques (stent retriever, aspiration or a combination of both). Flow in the occluded artery was completely or partially restored in all cases. The clinical outcome ranged from complete recovery (without neurological deficits) to a coma (a fatal outcome).

We present a case of a 77-year-old female patient with a medical history of hypertension, diabetes

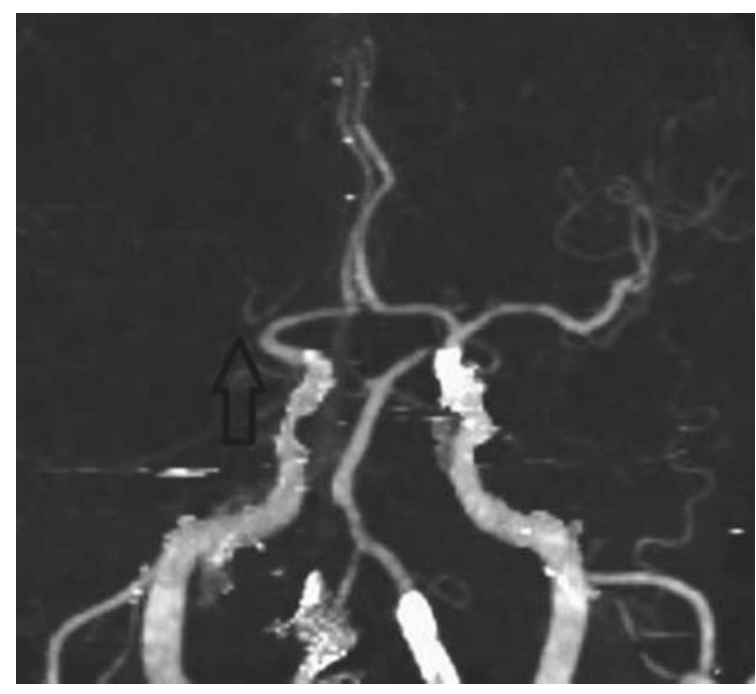

FIGURE 1. Initial imaging workup upon arrival at the general hospital. CT angiography (CTA) shows right M1 occlusion (arrow).

mellitus type 2, hyperlipidaemia and atrial fibrillation who was admitted to the general hospital with an acute onset of left-sided hemiplegia and dysarthria (National Institutes of Health Stroke Scale [NIHSS]: 4, Modified Rankin Scale [mRS]: 3). The patient was last seen without deficits 80 minutes prior to admission. The initial computed tomography (CT) scan revealed no ischaemic brain damage, and CT angiography showed right M1 MCA occlusion (Figure 1). Intravenous thrombolysis (IVT) was administered after 173 minutes and discontinued due to the sudden loss of conscience, the deviation of the head toward the left side and tonic-clonic spasms of the left extremities. The control CT scan performed under general anaesthesia

TABLE 1. Comparing 6 reported cases of mechanical thrombectomy in acute bilateral ICA and/or MCA occlusions

\begin{tabular}{|c|c|c|c|c|c|c|}
\hline \multirow{2}{*}{$\begin{array}{l}\text { Author, (Year), } \\
\text { reference }\end{array}$} & \multirow{2}{*}{ Clinical presentation } & \multirow{2}{*}{$\begin{array}{c}\text { Sex/age } \\
\text { (years) }\end{array}$} & \multicolumn{2}{|c|}{ Site of occlusion } & \multirow{2}{*}{$\begin{array}{l}\text { Mechanical } \\
\text { thrombectomy } \\
\text { (technique) }\end{array}$} & \multirow{2}{*}{$\begin{array}{l}\text { Clinical } \\
\text { outcome }\end{array}$} \\
\hline & & & ICA & MCA & & \\
\hline $\begin{array}{l}\text { Dietrich et al. } \\
(2014)^{5}\end{array}$ & $\begin{array}{l}\text { left hemiparesis, } \\
\text { progressing to coma }\end{array}$ & $M / 72$ & - & $+(\mathrm{Ml})$ & aspiration+stent- retriever & minor deficit \\
\hline $\begin{array}{l}\text { Pop et al. } \\
(2014)^{6}\end{array}$ & $\begin{array}{l}\text { impaired } \\
\text { consciousness }\end{array}$ & $\mathrm{F} / 78$ & + & $+(M 2)$ & stent-retriever & no deficit \\
\hline $\begin{array}{l}\text { Pop et al. } \\
(2014)^{6}\end{array}$ & right sided weakness & $F / 66$ & + & $+(M 1)$ & stent-retriever & severe deficit \\
\hline $\begin{array}{l}\text { Braksick et al. } \\
(2018)^{7}\end{array}$ & coma & $\mathrm{F} / 76$ & - & $+(M 1)$ & - (no data) & coma \\
\hline $\begin{array}{l}\text { Larrew et al. } \\
(2019)^{8}\end{array}$ & coma & $\begin{array}{l}\text { - (no } \\
\text { data) / } \\
\text { middle } \\
\text { age }\end{array}$ & + & +- & aspiration & fatal \\
\hline $\begin{array}{l}\text { Storey et al. } \\
\text { 2019) }\end{array}$ & $\begin{array}{l}\text { hemiparesis / } \\
\text { hemiplegia }\end{array}$ & $F / 64$ & + & $+(M 1, M 2)$ & aspiration+stent-retriever & minor deficit \\
\hline
\end{tabular}

$F=$ female $; I C A=$ internal carotid artery; $M=$ male; $M C A=$ middle cerebral artery 

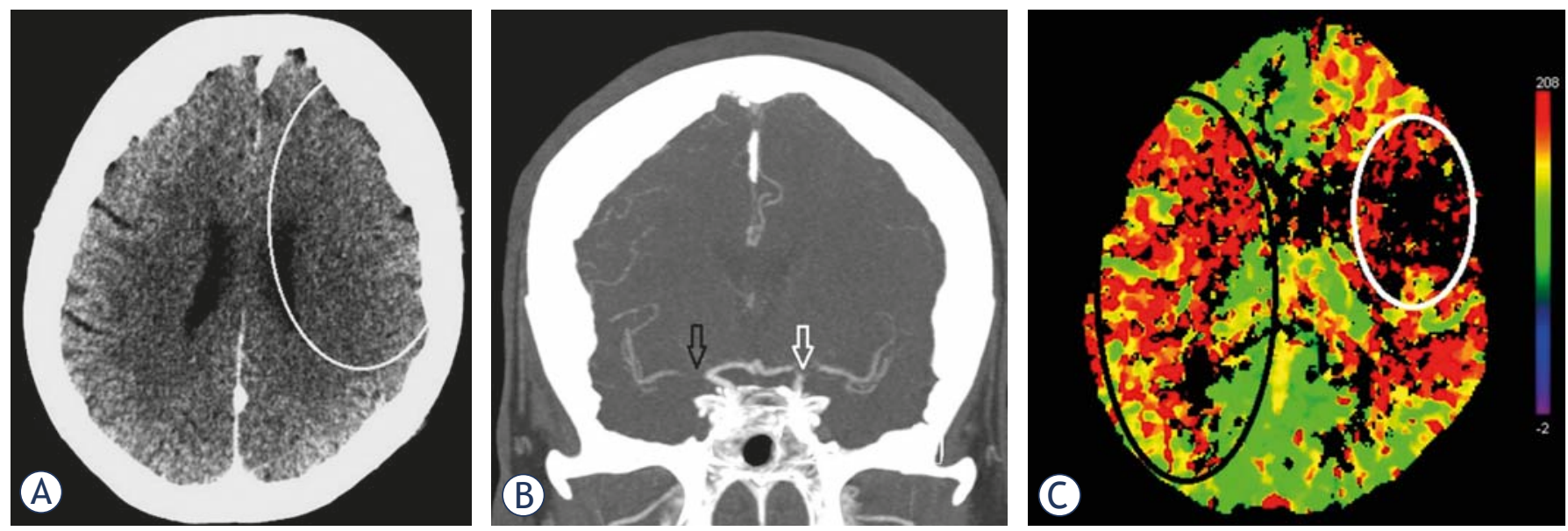

FIGURE 2. (A) Control images taken in the general hospital after clinical deterioration during intravenous thrombolysis and before the transfer to the tertiary institution. There were still no signs of ischaemic brain damage in the right cerebral hemisphere but there were subtle signs of stroke in the left middle cerebral artery (MCA) territory (white line delineates loss of cortical grey matter - white matter differentiation in the frontoparietal lobe with sulcal effacement). (B) Control images taken in the general hospital after clinical deterioration during intravenous thrombolysis and before the transfer. CT angiography (CTA) showed persistent right Ml occlusion (black arrow) but also left carotid "T" occlusion (white arrow). (C) Control images taken in the general hospital after clinical deterioration during intravenous thrombolysis and before the transfer. CT perfusion imaging (CTP) showed a penumbra in the right MCA territory (black circle) and irreversible brain damage in the left MCA territory (white circle).

showed no haemorrhagic complications. Since it was assumed that the patient had a symptomatic epileptic seizure, IVT was continued. The sedated patient was immediately transported to a tertiary institution, where a multimodal CT protocol (native $\mathrm{CT}$ scan, $\mathrm{CT}$ angiography [CTA] and CT perfusion imaging [CTP]) was performed, and the results revealed no signs of ischaemic brain damage in the symptomatic right cerebral hemisphere despite the presence of M1 occlusion and subtle (newly appeared) signs of irreversible brain damage in the contralateral MCA territory due to left ICA ("T") occlusion. It was obvious that an additional embolic occlusion occurred during the IVT treatment. Although the time of occlusion was long, the CTP imaging results showed a penumbra in the right MCA territory and not in the contralateral left MCA territory, where irreversible brain damage occurred within 3 hours (Figure 2).

Conventional (digital subtraction) angiography confirmed right MCA occlusion with good collateral flow provided by the right anterior cerebral artery (ACA). However, the situation on the left side had changed. Namely, a complete recanalization of the carotid " $\mathrm{T}$ " occlusion was seen angiographically as a consequence of thrombolysis and distal migration of the thrombembolus (now presenting with proximal M2 segment occlusion of the major MCA branch). However, no collateral flow was observed despite complete left ACA patency
(Figure 3). Endovascular mechanical recanalization with aspiration was successfully performed on the right side (Figure 4A). The same procedure was not performed on the left side because there was irreversible brain damage in the whole occluded arterial territory and a high risk of haemorrhagic complications. The postprocedural thrombolysis in cerebral infarction (TICI) scores were 3 (right MCA) and $2 b$ (left MCA).

The control CT and magnetic resonance imaging (MRI) scans performed on the next and subsequent days revealed no ischaemic damage on the right side and acute ischaemic stroke in the corresponding left MCA territory (Figure 4B). The patient was discharged with an improved clinical condition. However, severe neurological deficits (global dysphasia, dysarthria, spastic hemiplegia of the right limbs) were a consequence of permanent brain damage on the left side (NIHSS 5, mRS 5).

\section{Discussion}

To the best of our knowledge, there are only six reports about mechanical thrombectomy being performed for acute bilateral ICA and/or MCA

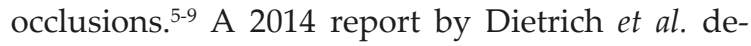
scribes two M1 MCA thrombectomy procedures being performed in sequence. Aspiration was attempted without success, so multiple passes with 
stent retrievers were performed for successful recanalization. ${ }^{5}$ Another report from the same year (2014) by Pop et al. demonstrates two cases for which ICA-MCA (M1, M2) bilateral thrombectomy procedures with stent retrievers were successful. ${ }^{6}$ A recent report by Larrew et al. (2019) describes a novel successful method for bilateral ICA occlusion, simultaneous recanalization, which utilizes two interventionalists and technicians simultaneously for aspiration thrombectomy. ${ }^{8}$ In the latest report by Storey et al. (2019), sequential M1-M2 MCA mechanical thrombectomy was successfully performed using a combination of the stent retriever and aspiration techniques. ${ }^{9}$ A case of stroke similar to that described in our study was reported by Braksick et al. (2018), where M1 MCA occlusion also occurred during the thrombolytic treatment of contralateral M1 MCA occlusion. Despite attempts at clot retrieval, flow was not completely restored, and the patient remained comatose. ${ }^{7}$

As so few related cases have been reported, it is unclear whether the outcomes can be improved by the optimization of the assessment and endovascular approach..$^{10} \mathrm{~A}$ comparison of our case with the abovementioned cases shows that the initial clinical presentations are similar, as they are cases of rapid deterioration with a loss of conscience. A prompt, adequate workup and treatment (a successful combination of standard care and mechanical recanalization) have led to clinical improvements and minimize morbidity and mortality. ${ }^{9}$ However, the clinical outcomes reported in the literature are still diverse..$^{5-9}$ It is unclear whether simultaneous thrombectomy, as described by Larrew et al. ${ }^{8}$, can improve the outcome by means of faster recanalization. The answer can possibly be found in our case study. Namely, an important conclusion from our case study is that the collateral capacity was more important than the time from stroke onset to successful flow restoration. It is well known that good collateral circulation in acute stroke patients is associated with better clinical and functional outcomes. ${ }^{11}$ The results of the DAWN trial show that thrombectomy plus standard care compared to standard care alone, even 6 to 24 hours after acute ischaemic stroke, yields better functional improvements in people with mismatch between clinical deficit and infarction. ${ }^{12}$ It is becoming obvious that the presence of the collateral flow, which defines the minimal blood flow in the penumbra, is equally important as the time in stroke patients being assessed for IVT. ${ }^{13}$ The clinical outcome in our patient with bilateral stroke during the same thrombolytic time window therefore confirms the importance
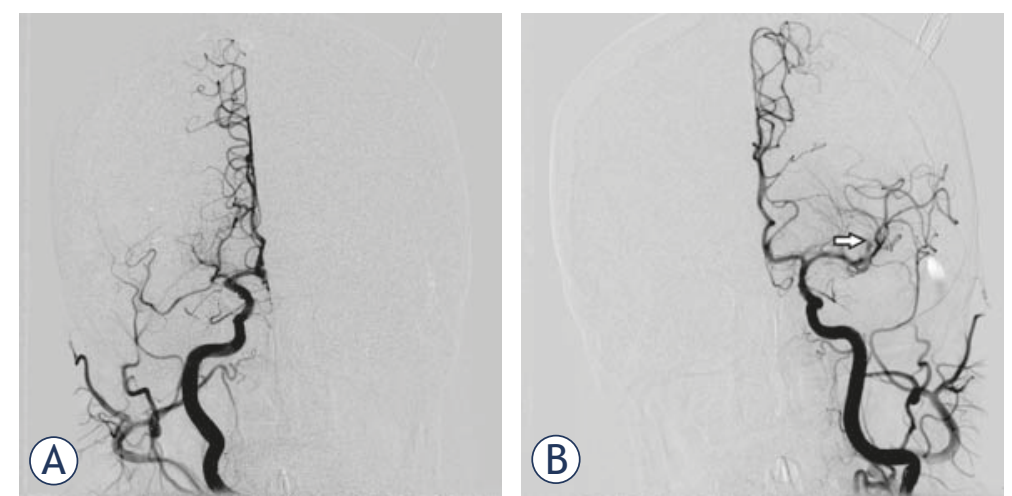

FIGURE 3. (A) Digital subtraction angiography (DSA) at the beginning of mechanical recanalization. Right internal carotid contrast injection confirming right $\mathrm{Ml}$ occlusion. (B) DSA at the beginning of mechanical recanalization. Left side contrast injection showing complete spontaneous recanalization of the carotid "T" occlusion with thrombembolar distal migration (occlusion of the proximal M2 segment of the major MCA branch) (arrow).

of collaterals. Namely, the region within the brain with good collateral circulation showed a complete recovery after mechanical recanalization at 7 hours after stroke onset, while the other (contralateral) region without collateral circulation exhibited a poor outcome no more than 3 hours after stroke onset. Our case shows that even in the same individual, the presence of collateral circulation varies across regions. Namely, the affected brain territory, even with comparable anatomic vascular architecture (patent ACA with possible collateral inflow) as the contralateral territory, exhibited shorter time from onset of occlusion to recanalization (with distal thrombus migration) but did not exhibit collaterals, leading to an immediate and irreversible inju-
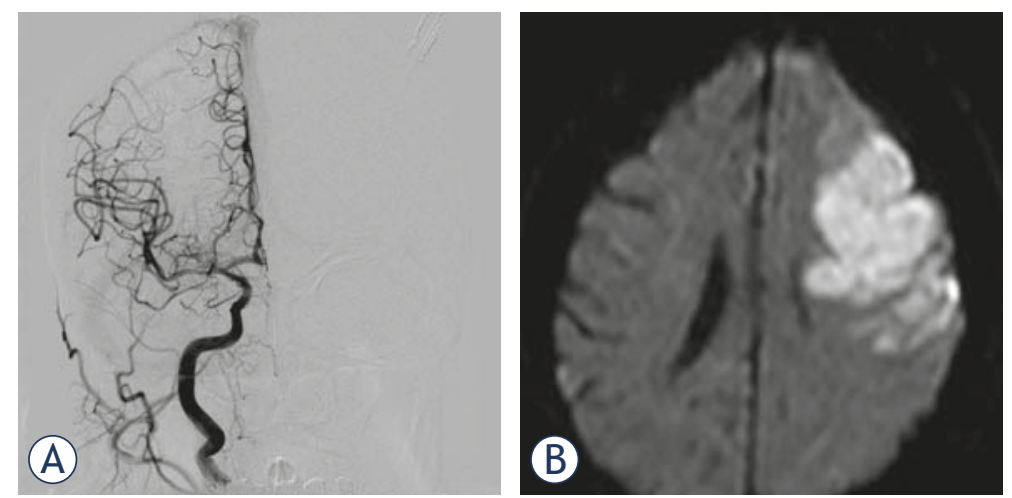

FIGURE 4. (A) Digital subtraction angiography (DSA) after mechanical recanalization. Right Ml mechanical recanalization (aspiration device) led to complete flow restoration. (B) MR diffusion weighted imaging (DWI) scan taken 6 days after mechanical recanalization: complete salvage of the affected right middle cerebral artery (MCA) brain parenchyma (recanalization at 7 hours after stroke onset). In contrast, subsequent persistent left $\mathrm{M} 2$ occlusion without collateral flow resulted in significant stroke within 3 hours after stroke onset. 
ry. To the best of our knowledge, we presented the first case of subsequent contralateral large cerebral artery (MCA-ICA/MCA) occlusion during IVT, where the outcome after standard care and mechanical thrombectomy was not dependent on the time from stroke onset but rather on the capacity of collateral circulation exclusively.

Simultaneous thrombectomy, as described by Larrew et al. ${ }^{8}$, offers an efficient and feasible means to reduce the time to recanalization. However, our case study confirms the fact that the outcome is still very much dependent on the capacity of collateral circulation. The main drawback of simultaneous thrombectomy (compared to subsequent thrombectomy) is that it requires two neurointerventionalists and technicians (nurses). The procedure is technically challenging, and the team must be methodical and organized and communicate effectively to allow effective, efficient, simultaneous and safe progression on both sides. ${ }^{10}$ It is likely that simultaneous thrombectomy would not have any impact on the outcome in our case study of a patient with poor collaterals. Interestingly, the TICI scores for the patient in our study were the same as those reported for patients treated with simultaneous thrombectomy in previous study (TICI 3 for one side and TICI $2 \mathrm{~b}$ for contralateral side). The presence of collateral capacity, the location of the residual occlusion (MCA vs ACA-MCA), and a concomitant disease (better cardiac function) were key determinants of better outcomes in the patient in our study.

Understanding the importance of collaterals and preprocedural imaging with techniques that enable collateral flow assessment is becoming extremely important. Multiphase CT angiography (CTA), which enables the evaluation of collateral circulation within a single contrast injection, is a simple example. ${ }^{14}$ It is already accepted that for good outcomes, the onset-to-reperfusion time window should be adjusted according to the collateral status. ${ }^{15}$ In the future, perhaps a pretreatment imaging assessment of the thrombus itself will make stroke treatment planning easier. ${ }^{16}$ However, it seems that even in the era of mechanical recanalization, bilateral stroke is a severe condition with unpredicted outcomes.

\section{Conclusions}

Acute bilateral cerebral (ICA and/or MCA) occlusion leads to sudden severe neurological deficits (comas) with unpredicted prognoses, even in the era of mechanical recanalization.
As a the collateral capacity seems to be more important than the absolute time to flow restoration in determining the outcomes, simultaneous thrombectomy by itself probably does not lead to improved functional outcomes.

Because there are only a few reported cases, additional experience is needed to fully understand the outcomes of sequential and simultaneous thrombectomy.

\section{References}

1. Kwon SU, Lee SH, Kim JS. Sudden coma from acute bilateral internal carotid artery territory infarction. Neurology 2002; 58: 1846-9. doi: 10.1212/ wnl.58.12.1846

2. Hu WT, Wijdicks EF. Sudden coma due to acute bilateral M1 occlusion. Mayo Clin Proc 2007; 10: 1155. doi: 10.4065/82.10.1155

3. Zubkov AY, Klassen BT, Burnett MS, Rabinstein AA. Bilateral internal carotid artery occlusions resulting in near total acute brain infraction. Neurocrit Care 2007; 7: 247-9. doi: 10.1007/s12028-007-0076-y

4. Nawashiro H, Wada K, Kita H. Decerebrate posture following bilateral middle cerebral artery occlusion. Intern Med 2011; 50: 2063. doi: 10.2169/ internalmedicine.50.5843

5. Dietrich U, Graf T, Schäbitzb WR. Sudden coma from acute bilateral M1 occlusion: successful treatment with mechanical thrombectomy. Case Rep Neurol 2014; 6: 144-8. doi: 10.1159/000362160

6. Pop R, Manisor M, Wolff V, Habashy $M$, Rouyer $O$, Kehrli $P$, et al. Endovascular treatment in two cases of bilateral ischemic stroke. Cardiovasc Intervent Radiol 2014; 37: 829-34 doi: 10.1007/s00270-013-0746-4

7. Braksick SA, Robinson CP, Wijdicks EFM. Bilateral middle cerebral artery occlusion in rapid succession during thrombolysis. Neurohospitalist 2018; 8: 102-3. doi: 10.1177/1941874417712159

8. Larrew T, Hubbard Z, Almallouhi E, Banerjee C, Moss M, Spiotta AM Simultaneous bilateral carotid thrombectomies: a technical note. Oper Neurosurg 2019; pii: opz230. doi: 10.1093/ons/opz230

9. Storey C, Lebovitz J, Sweid A, Tjoumakaris S, Gooch R, Rosenwasse $\mathrm{RH}$, Jabbour P. Bilateral mechanical thrombectomies for simultaneous MCA occlusions. World Neurosurg 2019; 132: 165-8. doi: 10.1016/j. wneu.2019.08.236

10. Srivatsan A, Kan P. Commentary: simultaneous bilateral carotid thrombec tomies: a technical note. Oper Neurosurg 2019. pii: opz273. doi: 10.1093/ ons/opz273.

11. Bang OY, Goyal M, Liebeskind DS. Collateral circulation in ischemic stroke: assessment tools and therapeutic strategies. Stroke 2015; 46: 3302-9. doi: 10.1161/STROKEAHA.115.010508

12. Nogueira RG, Jadhav AP, Haussen DC, Bonafe A, Budzik RF, Bhuva P, et al Thrombectomy 6 to 24 hours after stroke with a mismatch between deficit and infarct. N Engl J Med 2018; 378: 11-21. doi: 10.1056/NEJMoa1706442

13. Bivard A, Spratt N, Miteff F, Levi C, Parsons MW. Tissue is more important than time in stroke patients being assessed for thrombolysis. Front Neurol 2018; 9: 41. doi: 10.3389/fneur.2018.00041

14. Garcia-Tornel A, Carvalho V, Boned S, Flores A, Rodríguez-Luna D, Pagola $\mathrm{J}$, et al. Improving the evaluation of collateral circulation by multiphase computed tomography angiography in acute stroke patients treated with endovascular reperfusion therapies. Interv Neurol 2016; 5: 209-17. doi $10.1159 / 000448525$

15. Kim BM, Beak JH, Heo JH, Nam HS, Kim YD, Yoo J, et al. Collateral status affects the onset-to-reperfusion time window for good outcome. J Neurol Neurosurg Psychiatry 2018; 89: 903-9. doi: 10.1136/jnnp-2017-317627

16. Vidmar J, Bajd F, Milosevic ZV, Kocijancic IJ, Jeromel M, Sersa I. Retrieved cerebral thrombi studied by $\mathrm{T} 2$ and ADC mapping: preliminary results. Radio Oncol 2019; 53: 427-33. doi: 10.2478/raon-2019-0056 